\section{Cyclic Plasticity of Pure Metals}

\author{
A. T. Winter, Cambridge \\ Cavendish Laboratory
}

Fatigue failures, even in the most ductile metals and alloys, occur by propagation of sharp "fatigue cracks", superficially similar to brittle cracks. Material at the tip of a fatigue crack will normally undergo cyclic plastic straining as a result of rather complicated Joadings of variable amplitude, and its response to this cyclic straining may well control propagation of the crack. However, this need not always be true and some authors have proposed mechanisms of crack extension which do not involve cyclic plasticity at all. Here we are concerned with cyclic plasticity as such, which we define as the response of a material to a cyclic plastic strain. The discussion will be limited to pure metals and simple conditions in which either the uniaxial plastic strain amplitude or stress amplitude are held constant. In tests on a single crystal, we are limited to the period before the fatigue crack has become large enough to affect the cross-section appreciably, but this permits us nevertheless, to study all but the final few percent of the fatigue life.

\section{Cyclic Hardening and Softening}

In a single crystal of a pure metal such as copper, the stress required to move a straight dislocation is extremely low. Consequently, in the absence of grain boundaries and impurities, the mechanical properties are entirely controlled by the dislocation microstructure. At the start of a fatigue test, an annealed single crystal is very soft, but as cyclic straining at a constant plastic strain amplitude proceeds, the dislocation density increases and the crystal hardens, so causing the stress amplitude to increase. This "cyclic hardening"

Fig. 2 - The 'two-phase' model for cyclic deformation. The section $A B$ of the cyclic stress/ strain curve is unstable and the experimentally observed curve exhibits a plateau in which slip bands occupy part of the crystal. This is contrasted with the plateau $D E$ for the reversible phase change between liquid and vapour.

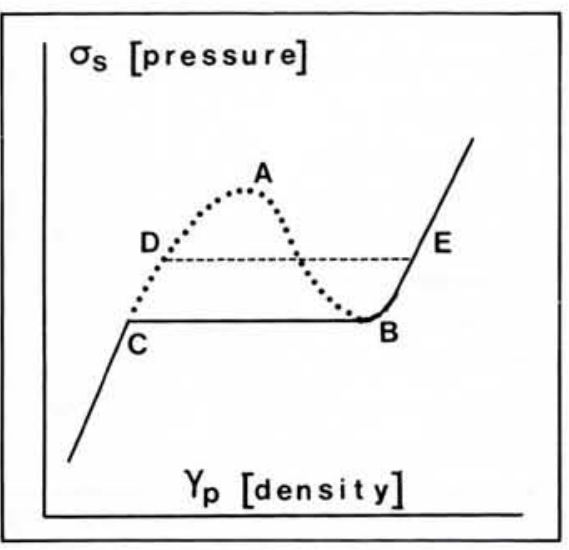

has the additional effect of keeping the distribution of strain reasonably uniform throughout the crystal, for if the strain amplitude increases locally, then the cyclic hardening rate will also increase locally and this will provide negative feedback.

If at the start of a fatigue test the crystal is in a heavily work-hardened condition, then cyclic straining may cause the dislocation microstructure to soften (Fig. 1).

\section{Saturation}

After perhaps a hundred or a thousand cycles of cyclic hardening, the stress amplitude ceases to rise. In many materials, a long period at an almost constant stress amplitude follows and is concluded only by the final (rapid) fatigue failure at $10^{5}$ - $10^{6}$ cycles. This saturation of cyclic hardening allows the possibility that macroscopic slip bands may form. Control of the strain distribution passes from the cyclic hardening curve to the cyclic stress/ strain curve, a plot of the saturated stress amplitude vs. plastic strain amplitude. So long as this curve has a strong upward gradient, the strain will remain more or less uniformly distributed, but if the cyclic stress/strain curve has a negative gradient, then the crystal normally will break up into active and inactive regions. There is an analogy here with the behaviour of a closed liquid-vapour system near its vapour pressure. The inactive regions of the specimen are analogous to the vapour (low density) whilst the active slip bands are analogous to the liquid (high density). Provided that the slip bands are free to deform independently of the surrounding "matrix" we may write a simple law of mixtures for the average strain amplitude in the specimen.

$$
\gamma_{\mathrm{p}}=f \gamma_{\mathrm{b}}+(1-f) \gamma_{\mathrm{m}} .
$$

Here $\gamma_{p}$ represents the average plastic strain amplitude in the crystal; $\gamma_{\mathrm{b}}$ and $\gamma_{\mathrm{m}}$ refer to the slip bands and matrix respectively; $f$ represents the volume fraction occupied by slip bands. Measurement of $f$ as a function of $\gamma_{p}$ yields values for $\gamma_{b}$ and $\gamma_{m}$ and for copper there is reasonable agreement that $\gamma_{\mathrm{b}} \sim 7.5 \times 10^{-3}$ and $\gamma_{\mathrm{m}} \leq 6 \times$ $10^{-5}$.

This two-phase model predicts a plateau in the cyclic stress/strain curve (Fig. 2) and this is in good agreement with experiment. Different authors report slightly different levels for the plateau but it seems to lie in the range $25-30 \mathrm{MPa}$.

\section{Tests at Constant Stress Amplitude}

Applied to tests at constant stress amplitude, the two-phase model predicts the existence of a critical value of stress amplitude, analogous to the saturated

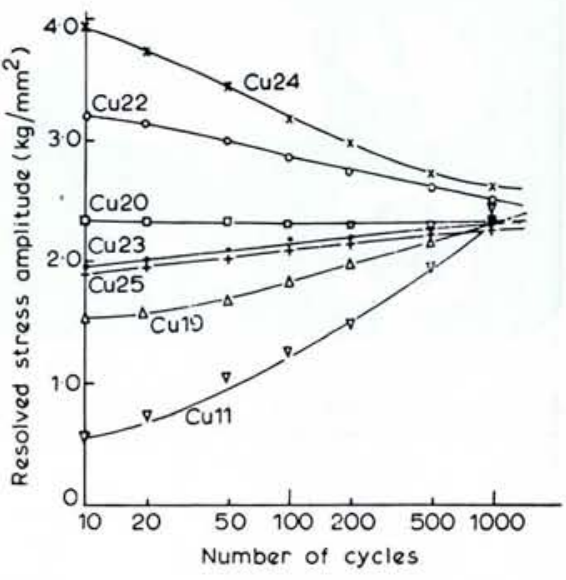

Fig. 1 - Cyclic hardening and softening curves for copper at $\gamma_{p} \sim 3 \times 10^{-3}$. The initial state of the crystal determines the subsequent behaviour, but all specimens approach the same saturated stress amplitude.

vapour pressure, below which fatigue hardening can continue indefinitely: the plastic strain amplitude then will decrease effectively to zero and the crystal will have an effectively infinite fatigue life. Above the critical stress amplitude, slip bands will propagate to fill the crystal $(f=1)$ : the strain amplitude will then be high and fatigue failure will be rapid. This behaviour is in good agreement with experiment including a reasonable similarity between the critical stress amplitude (known as the fatigue limit) and the level of the plateau.

\section{Dislocation Microstructures}

The matrix and slip bands contain markedly different dislocation microstructures (Fig. 3) which are responsible for their markedly different mechanical properties. After saturation the plastic strain amplitude in the matrix is very small $\left(\leq \gamma_{b} / 100\right)$. Therefore we can conclude that:

(i) Heavily dislocated regions in the matrix structure do not deform plastically;

(ii) The widths of the dislocation-free channels are so small that the Orowan stress is high enough to prevent plastic deformation here also.

This is not to say that an increased stress amplitude would not cause plastic deformation in the matrix structure, as occurs immediately after an increase in the plastic strain amplitude or during an "overshoot" in the applied strain.

The plastic strain amplitude in the slip bands is high $\left(\sim 7.5 \times 10^{-3}\right)$ so we may conclude that these contain a softer structure, consistent with the smaller volume fraction of heavily dislocated material and with the rather wider channels between the walls (spacing $\sim 1.4 \mu \mathrm{m}$ ).

The lack of observable lattice rotations in either structure implies that they do not contain any large preponderance of one sign of dislocation over the other. This is in contrast to the structures of mis-oriented cells sometimes found in fatigue and common in unidirectionally deformed speci- 


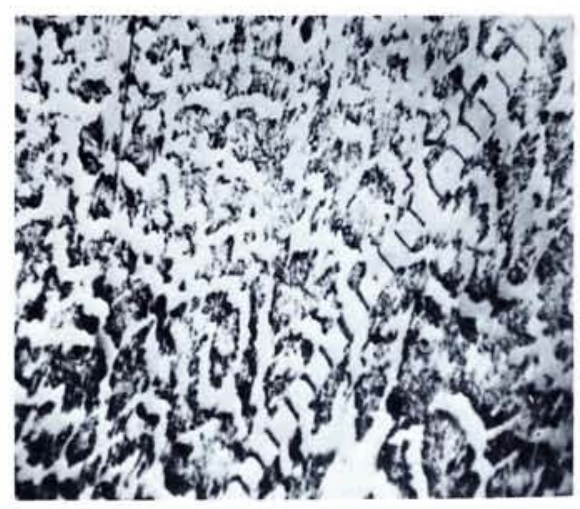

Fig. 3 - The soft 'wall structure' and the hard 'matrix structure' in copper fatigued to saturation; (above) the cross-slip plane and (right) the primary slip plane.

mens where the dislocation walls behave like low angle grain boundaries. At low strain amplitudes, both wall and matrix structures are composed almost entirely of primary dislocations.

Various other types of dislocation structure are observed in cyclically deformed metals, particularly in polycrystals, but their effects on the mechanical properties are still not clear. There is no satisfactory explanation for the regular spacing of 1.4 $\mu \mathrm{m}$ in the wall structure.

\section{Irreversibility of the Phase Change}

The liquid-gas phase change is reversible and a reduction of density causes some liquid to vaporise. This is not the case with the matrix-slip band system. It is certainly possible for the matrix structure to change into the wall structure and this does indeed happen, for example if the plastic strain amplitude is increased after saturation. The converse however, conversion of wall structure to matrix, has never been observed even after marked reductions in strain amplitude. This irreversibility has obvious consequences for cyclic deformation at variable amplitudes.

\section{Validity of the Two Phase Model at High Cumulative Strain}

Slip bands form from the matrix around saturation (perhaps after 1000 cycles) and their subsequent rate of propagation is rapid, producing a sizeable volume fraction rather quickly. Thus Winter was able to measure the volume fraction in order to test the two phase model at 15000 cycles. Mughrabi and Finney and Laird working at comparable cumulative strains obtained similar results. This rapid rate of propagation is not maintained for long, as can be established simply by remarking that after fatigue failure at, say, $2 \times 10^{5}$ cycles, the crystal is not completely filled by slip bands. On the least favourable assumptions, the propagation rate must decrease by at least an order of magnitude after saturation. But the experimental observation can be stated more strongly, for mea-

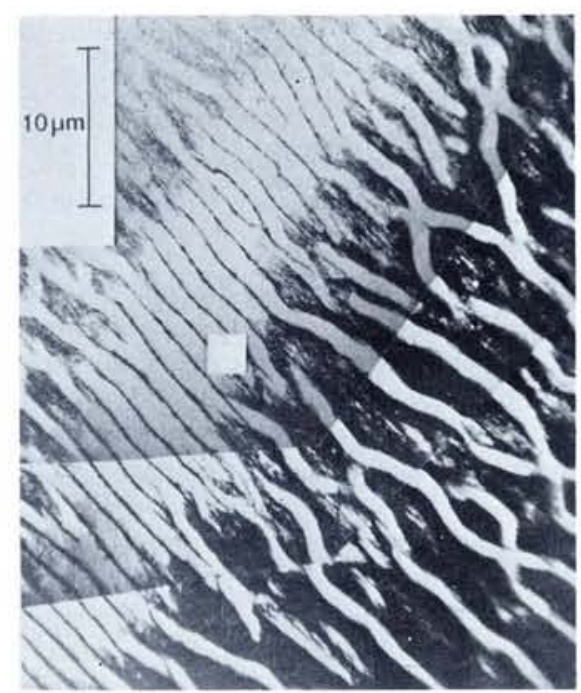

surements of $f$ on crystals which have suffered fatigue failure yield results which are not significantly different from those obtained at 15000 cycles. We may thus look for a decrease of 2-3 orders of magnitude in the propagation rate after saturation even though microscopical studies suggest that some slow propagation does indeed continue after saturation.

We may also ask how long the slip bands can operate without becoming inactive. In magnesium, for example, saturation is terminated by a period of renewed cyclic hardening. The two phase model has not been established in this case but could still apply if the slip bands had a finite active life. As old slip bands became inactive, new ones would form in accord with the two phase model. This could continue until the crystal was full of slip bands and then some other mechanism would need to take over, presumably at a higher stress amplitude. This does not seem to be an important problem in copper in normal circumstances, but if the fatigue life could be prolonged (for example by periodic electropolishing) it might become significant. We can already say that the deformation mechanisms in the slip bands are extraordinarily reversible in the sense that they do not lead to cumulative damage. If a slip band remains active for $10^{5}$ cycles then it has undergone a cumulative plastic strain of around 3000 .

Direct Measurement of $\gamma_{b}$ and $\gamma_{m}$ Using Optical Interferometry

Finney and Laird and Z.S. Basinski (private communication) have used optical interferometry to study the local distribution of strain directly. In summary their results show (Fig. 4):

(i) After saturation there is no measurable plastic strain in the matrix;

(ii) The average plastic strain in the slip bands is in accord with the two phase model described above; BUT

(iii) The plastic strain in the slip bands themselves is not uniformly distributed, nor is it the same from one slip band to another; (iv) There exist narrow slip bands in which the local shear strain may be as high as unity. (These are observed as discrete steps on the specimen surface.)

The interferometry is performed only over a rather small number of cycles, after repolishing the crystal, which leaves open the possibility that the average mentioned in (ii) might be taken for a given slip band over a few hundred cycles. Indeed the observed irreversibilities in strain within the slip bands demonstrate that some such averaging is required.

These results are obviously of crucial importance in considering how the dislocations within a slip band achieve the cyclic plastic strain.

\section{Cyclic Deformation of Polycrystals}

The two phase model has been established for copper polycrystals, at least for large grain sizes.

In order to produce a plateau in the cyclic stress-strain curve, the slip bands must be able to undergo plastic straining independently of the matrix. In single crystals, this does not present a problem because the slip bands can take the favourable form of narrow slabs approximately parallel to the crystallographic shear planes. In polycrystals, the situation is less clear cut, especially in the interior where plastic shear strains in different grains must be made compatible with each other. The stresses produced are not impossibly large only because the plastic strains involved in fatigue are rather small. For grain sizes greater than, say, 100 $\mu \mathrm{m}$ the two phase model may predict a plateau, but for smaller grain sizes this does not seem likely. Because it involves plastic shear on only a single crystallographic plane, the two phase model predicts that the cyclic stress/strain curve will be very seriously affected by the texture of a polycrystal.

The wall and matrix structures have been observed in the interior grains of polycrystals along with other dislocation structures not commonly observed in single crystals. Thus slip bands can be said to exist in the interior grains. However the processes by which a slip band becomes a small fatigue crack seem to be confined to the surface of the specimen.

Fig. 4 - Optical interferogram from a copper crystal fatigued to saturation, electropolished and then given $10 \% / 4$ cycles. (Courtesy of Finney and Laird).

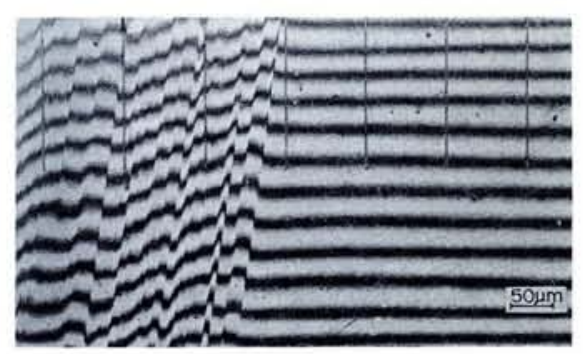

